\title{
Immature Myeloid Cell
}

National Cancer Institute

\section{Source}

National Cancer Institute. Immature Myeloid Cell. NCI Thesaurus. Code C113503.

Hematopoietic cells that express the common myeloid marker CD33 but do not express the MHC class II molecule HLA-DR or other markers of mature myeloid or lymphoid cells. An accumulation of these cells may be associated with a decreased number of dendritic cells in the peripheral blood of patients with head and neck, lung or breast cancer. 